\title{
Resveratrol preserves cerebrovascular density and cognitive function in aging mice
}

\section{Charlotte A. Oomen ${ }^{1}$, Eszter Farkas ${ }^{2}$, Viktor Roman ${ }^{1}$, Eline M. van der Beek ${ }^{3}$, Paul G. M. Luiten and Peter Meerlo'}

\author{
Department of Molecular Neurobiology, Center for Behavior and Neuroscience, University of Groningen, Groningen, The Netherlands \\ 2 Department of Physiology, School of Medicine, University of Szeged, Szeged, Hungary \\ ${ }^{3}$ Danone Research, Centre for Specialised Nutrition, Wageningen, The Netherlands
}

\section{Edited by:}

Jean Mariani,

Universite Pierre et Marie Curie, France

Reviewed by:

Karl Herrup, Case Western University, USA

\section{${ }^{*}$ Correspondence:}

Charlotte A. Oomen,

SILS - Center for Neuroscience,

University of Amsterdam, Science Park

904, 1098 XH Amsterdam,

The Netherlands.

e-mail:c.a.oomen@uva.nl

Resveratrol, a natural polyphenol abundant in grapes and red wine, has been reported to exert numerous beneficial health effects. Among others, acute neuroprotective effects of resveratrol have been described in several models of neurodegeneration, both in vitro and in vivo. In the present study we examined the neuroprotective effects of long-term dietary supplementation with resveratrol in mice on behavioral, neurochemical and cerebrovascular level. We report a preserved cognitive function in resveratrol-treated aging mice, as shown by an enhanced acquisition of a spatial Y-maze task. This was paralleled by a higher microvascular density and a lower number of microvascular abnormalities in comparison to aging non-treated control animals. We found no effects of resveratrol supplementation on cholinergic cell number or fiber density. The present findings support the hypothesis that resveratrol exerts beneficial effects on the brain by maintaining cerebrovascular health. Via this mechanism resveratrol can contribute to the preservation of cognitive function during aging.

Keywords: resveratrol, antioxidant, French paradox, aging, maze learning, cholinergic system, cerebrovascular system microvessels

\section{INTRODUCTION}

Resveratrol (trans-3,5,4'-trihydroxystilbene) is a natural polyphenol, enriched in grapes and red wine, with potential beneficial effects on human health. In fact, the lower incidence of cardiovascular disease in wine drinking countries despite a diet high in saturated fat, a phenomenon referred to as the 'French paradox', has been associated with the actions of resveratrol (Renaud and de Lorgeril, 1992; Kopp, 1998; Wu et al., 2001).

Resveratrol exerts a myriad of physiological effects (for reviews see Fremont, 2000; Baur and Sinclair, 2006) including antioxidant (Frankel et al., 1993; Fremont et al., 1999; Mizutani et al., 2001; Jang and Surh, 2003), phytoestrogenic (Gehm et al., 1997), vasorelaxing (Fitzpatrick et al., 1993; Jager and Nguyen-Duong, 1999; Das et al., 2005), anti-inflammatory and anti-carcinogenic activity (Jang et al., 1997). In addition, resveratrol has been reported to extend life span in yeast (Howitz et al., 2003) and promote survival in mice on a high-caloric diet (Baur et al., 2006).

Besides effects on general health and life span, a number of in vivo studies suggest that injection of resveratrol during the induction of cerebral ischemia protects the brain against acute neurodegeneration (Wang et al., 2002; Lu et al., 2006). Also, dietary resveratrol supplementation was able to reduce ischemia-induced neuronal damage (Virgili and Contestabile, 2000; Dong et al., 2008). In hippocampal slices, cell death and altered neuronal responses due to oxygen and glucose deprivation were attenuated by resveratrol (Zhang et al., 2008). Considering its health and survival promoting effects and its neuroprotective potential after acute treatment in models of neurodegeneration, it is an intriguing question whether chronic dietary resveratrol could protect against slow age-related decline in brain function and cognitive performance in normal mice.
The process of aging affects the brain in multiple ways and causes a progressive decline in neuronal and cognitive function. One of the major underlying processes in brain aging is the deterioration of the cerebrovascular system, as it is often seen in age-related disorders and dementia (Buee et al., 1994; De Jong et al., 1997; Farkas et al., 2000; Farkas and Luiten, 2001; Riddle et al., 2003; Iadecola, 2004). Aging is associated with changes in microvascular plasticity and a reduced microvascular density (Amenta et al., 1995; Sonntag et al., 1997, 2000; Lynch et al., 1999). In addition, aging causes a progressive increase in microvascular abnormalities that affect blood-brain barrier function, including surface irregularities (Lee et al., 2000), thickening of the basement membrane and fibrous collagen deposits in the capillary wall (Burns et al., 1981; Keuker et al., 2000). Such abnormalities may decrease perfusion of the brain, leading to a decrease in nutrient and oxygen supply, which may be an important component in the development of age-related cognitive impairment (De Jong et al., 1997; Farkas et al., 2000, 2007; Farkas and Luiten, 2001; Riddle et al., 2003) and render the brain vulnerable to degenerative mechanisms. Therefore, the cerebrovascular system is an important target in preserving neuronal and cognitive health.

Since resveratrol has a potentially beneficial influence on several parameters involved in aging, including vascular function (Labinskyy et al., 2006) we here examine the neurobiological effects of long term, regular resveratrol intake in aged mice. We hypothesized that resveratrol preserves neuronal health and cognitive function in aging mice by delaying age-dependent cerebrovascular deterioration. In order to determine the effects of aging and long-term resveratrol administration on behavioral level, we 
measured general exploratory activity in an open-field test and analyzed learning and memory performance in a hippocampusdependent spatial Y-maze task. To assess cerebrovascular condition, we performed detailed structural analysis of cerebral microvessels in the cortex and hippocampus by means of electron microscopy. In addition, we characterized potential alterations in the cholinergic neurotransmitter system, a system often affected in age-related cognitive decline, by determining histochemically labeled cell numbers and fiber density.

\section{MATERIALS AND METHODS ANIMALS AND HOUSING}

The experiment was performed with male C57Bl/6 mice (Harlan, Horst, The Netherlands). The procedures concerning animal care and treatment were approved by the ethical committee for the use of experimental animals of the University of Groningen. Animals were individually housed under a 12 -h light/12-h dark cycle with lights on at 8:00 AM. Food and drinking water were provided ad libitum, except for the period of behavioral testing as described below. The experiment started with 60 mice, half of which served as control $(\mathrm{CON})$ and half of which received resveratrol in their food from the age of 1 year onwards (RES). Between the ages of 18 and 20 months, spatial learning performance of the animals was studied in a Y-maze test. At the age of 24 months, brains were collected and processed for histochemical analysis of cholinergic parameters and electron microscopic analysis of microvascular ultrastructure in the cortex and hippocampus.

\section{RESVERATROL SUPPLEMENTATION}

Resveratrol was provided in the food ( $150 \mu \mathrm{g}$ resveratrol/gram food; Hope Farms, Woerden, The Netherlands). To rule out differences in food intake between animals on a control diet and resveratrolsupplemented diet, food intake and body weight were monitored every 2 weeks. To establish whether resveratrol provided in the food enters the circulation, we performed a pilot experiment in which we collected blood from six mice by decapitation after 1 day and 30 days of supplementation. Plasma concentrations of resveratrol were measured (Danone Research, Wageningen, The Netherlands). After 1 day of supplementation, resveratrol levels in the plasma were $88.5 \pm 20.5 \mu \mathrm{g} / \mathrm{l}$ in the last hour of the dark or active phase, when mice consume most of their food, and $41.0 \pm 2.6 \mu \mathrm{g} / \mathrm{l}$ in the last hour of the light phase or resting phase ( $n=3$ each). After 1 month of supplementation, levels were $102.7 \pm 35.3 \mu \mathrm{g} / \mathrm{l}$ and $30.7 \pm 11.2 \mu \mathrm{g} / \mathrm{l}$ at the end of active phase and rest phase, respectively ( $n=3$ each). These measurements confirmed that resveratrol ingested via the food indeed entered the circulation and that levels appeared to fluctuate over the day in a fashion that paralleled food intake.

\section{OPEN-FIELD TEST}

To examine general exploratory activity, mice were subjected to an open-field test for $5 \mathrm{~min}$. The open field consisted of a circular arena with a diameter of $120 \mathrm{~cm}$ surrounded by a $60-\mathrm{cm}$ high wall. The arena was divided in two imaginary concentric zones, a central zone (60-cm diameter) and an outer zone (120-cm diameter). Position and locomotion of the mice were recorded and analyzed with a computerized video tracking system (Ethovision, Noldus Information Technology, Wageningen, The Netherlands). The number of visits, total time and distance spent in each of the two zones was measured and used to analyze exploration patterns and general activity. The open-field arena was thoroughly cleaned before a new animal was tested.

\section{Y-MAZE LEARNING}

To examine cognitive performance, the mice were subjected to a Y-maze test in which they had to learn the location of a food reward in one of two accessible choice arms (Havekes et al., 2007). The test was conducted in a tubular, transparent plexiglass Y-maze consisting of a start arm and two test arms forming the Y. All arms of the maze were $27.5 \mathrm{~cm}$ long, had a diameter of $5 \mathrm{~cm}$, and were at a $120^{\circ}$ angle from each other. The home cages of the mice were equipped with a small sliding door that connected to the starting arm. One of the two test arms was baited with a food reward consisting of small crumbs of the regular food. Food crumbs were also placed below perforations at the end of the other test arm to prevent animals from discriminating between baited and non-baited arms by olfactory cues. Small grey plastic blocks $(1 \mathrm{~cm}$ high) were placed $4 \mathrm{~cm}$ from the end of the arms to prevent visual inspection for food presence from a distance. A guillotine door halfway each arm could be operated manually from the experimenter's position and was used to allow animals only one choice in each training trial. The experimental room contained visual cues, which served as distal spatial cues. To assure sufficient motivation for the test, the animals were food restricted to about $85 \%$ of their original body weight. Food restriction started 3 days before the test. The animals received their food daily between 16:00 and 18:00 PM.

The Y-maze test took place during the first half of the light phase between 10:00 and 14:00 in a room adjacent to the home cage room. The animals were carried in their home cage to the experimental room and the cage was then connected to the Y-maze. On the first day of the test animals were habituated to the maze by allowing them to freely explore the arms for $5 \mathrm{~min}$. They further received two habituation trials during which they had access to the right arm only on the first trial and the left arm only on the second trial, with arms baited during each habituation trial. After the habituation day, mice received a daily training session consisting of eight trials consecutively. During the entire training phase, either the right or left arm was baited. This was constant for a given animal, but randomized between animals and treatments. When during a trial an animal visited one of the two accessible arms, the non-visited arm was closed. After the animal retreated to the start box, the start arm connected to the start box was blocked preventing re-entrance of the maze. After cleaning all arms with damped paper cloth, and re-baiting the same arm, the animal was again allowed to explore either the right or left test arm. A visit to the baited arm was recorded as a correct trial. Mice were trained in the Y-maze for 3 consecutive days and 10 days after the final training day, animals were subjected to another series of eight trials to test their memory.

\section{TISSUE PROCESSING}

At the age of 24 months animals were sacrificed for histochemical and electron microscopical analysis. Mice were anaesthetized by a high dose of sodium pentobarbital (Nembutal, $60 \mathrm{mg} / \mathrm{kg}$ bodyweight) and transcardially perfused with heparinized saline 
followed by $4 \%$ phosphate buffered paraformaldehyde. After perfusion, brains were carefully removed from the skull and kept in $0.01 \mathrm{M}$ PBS overnight. A random subset of 18 brains $(n=9$ for both CON and RES) were further processed for electron microscopy. Another random subset of 22 brains ( $n=11$ for both CON and RES) was used for histological analysis.

\section{HISTOLOGY}

For histology, brain tissue was cryoprotected in 30\% sucrose in $0.1 \mathrm{M}$ phosphate buffer (PB) for $48 \mathrm{~h}$ after which $20 \mu \mathrm{m}$ sections were cut with a cryostat (Leica CM3050). To analyze possible changes in cholinergic cells and fibers, an enzymatic staining for acetylcholinesterase (AChE) was performed in every 10th section. Sections were postfixed in a $2.5 \%$ glutardialdehyde solution in $\mathrm{PB}$ overnight at $4^{\circ} \mathrm{C}$. AChE histochemistry was carried out according to previously described methods (Hedreen et al., 1985). In brief, sections were incubated in an acetylthiocholine-iodide containing incubation buffer, followed by sodiumsulfide and finally intensified with a $0.1 \%$ silver nitrate solution. Cell bodies positive for AChE were manually counted by an experimenter blind for the treatment group in two representative sections per animal, containing the nucleus basalis (around bregma -0.7). Cell numbers were expressed as average number per section. AChE fiber density measurements were performed in two sections per animal by a computerized image analysis system (Quantimet, Leica). A contour was drawn around the stratum radiatum and stratum oriens of the CA1 region of the dorsal hippocampus in which fiber density was computed as a percentage of the total surface area covered by cholinergic fibers. The same procedure was used in the molecular layer of the dentate gyrus of the dorsal hippocampus and for two contours per section in the parietal cortex II between bregma -1.4 and -2.3 .

\section{ELECTRON MICROSCOPY}

After perfusion, 18 brains were prepared for electron microscopy (de Wilde et al., 2002; Farkas et al., 2003). Briefly, tissue blocks of the frontoparietal cortex and the dorsal hippocampus were incubated in an aqueous solution of $1 \% \mathrm{OsO}_{4}$ and $5 \% \mathrm{~K}_{2} \mathrm{Cr}_{4} \mathrm{O}_{7}$ (1:1) after thorough rinsing. Subsequently, the samples were dehydrated, incubated in $1 \%$ uranyl-acetate, and embedded in glycide ether. Semi-thin sections $(0.3 \mu \mathrm{m})$ were cut on an ultramicrotome and stained on object glasses with a 1:1 mixture of $1 \%$ ethylene blue and 1\% Azure II blue. The samples were then coverslipped with DPX and analyzed under a light microscope. Then, 90-nm ultra thin sections were cut of the same blocks and three non-serial sections were collected on 200 mesh copper grids. Subsequently, preparations were contrasted with 5\% uranylacetate and Reynolds lead-citrate solution. Finally, the samples were analyzed and photographs were taken with a Philips TM10 transmission electron microscope.

For quantitative analysis, approximately $4000 \mu \mathrm{m}^{2}$ tissue surface was scanned systematically in each region, and $61 \pm 10$ microvascular cross sections were examined in each sample. Vascular density was calculated for a standard surface area with the help of the sample grid as follows: the number of encountered microvascular profiles was divided by the examined surface area, which was determined by counting the number of grid squares of a standard size provided by the distributor. Vascular density was then expressed as the number of microvascular profiles on $1 \mathrm{~mm}^{2}$ surface. In addition to microvessel density, the occurrence of the following features were quantified: luminal endothelial protrusions, large empty endothelial vacuoles and basement membrane thickenings. The basement membrane was considered thickened when local exfoliations were observed in case of capillaries, or hyalinosis in case of arterioles. The number of microvessels displaying any of the noted abnormalities was counted and expressed as percentage of the total number of microvessels examined. Capillaries and arterioles were analyzed separately. Since arterioles were encountered at high enough number for statistical analysis only in the hippocampal vascular layer, quantitative analysis of arteriolar condition was performed for this region of the hippocampus only.

\section{STATISTICAL ANALYSIS}

Y-maze performance was statistically analyzed with a one way ANOVA paradigm for repeated measures. Open-field behavior, histochemical data and electron microscopical data from CON and RES animals showed equal variances as determined by Levene's test for equality of variance $(p>0.05)$ and there was no indication that the data was not normally distributed, as was determined by a one-sample Kolmogorov-Smirnov test $(p>0.05)$. Therefore, differences were statistically tested using an independent samples Student's $T$-test (2-tailed). To evaluate relationships between cerebrovascular parameters and Y-maze performance, a Pearson correlation analysis was applied. Data are expressed as averages \pm SEM, $p<0.05$ was considered as significant.

\section{RESULTS}

\section{SURVIVAL AND FOOD INTAKE}

Animals received resveratrol in their food from 1 year of age onwards. Adding resveratrol to the food did not affect food intake, and body weight (data not shown). Before behavioral testing started, at the age of 18-20 months, four animals had died (2 CON and 2 RES). After behavioral testing, but before the end of the experiment at 24 months, nine more animals died (4 CON and 5 RES). There were no significant differences in mortality rate between CON and RES mice, and regular visual inspection did not indicate obvious differences in general health either.

\section{BEHAVIORAL PERFORMANCE}

In the open-field test, CON and RES mice displayed the same amount of locomotion (total distance covered), however, the RES group spent about $50 \%$ more time in the center of the arena than the control animals ( $T$-test, $p=0.003$; Figures 1A,B).

Also during the habituation trial of the Y-maze, CON $(n=28)$ and RES $(n=28)$ mice displayed similar levels of activity in terms of arm visits and spontaneous alternations (data not shown). However, during the training phase, chronic resveratrol supplementation improved learning (repeated measures ANOVA for number of correct choices on day 1-3: $F_{1,54}=6.156, p=0.016$; Figure 2). A $T$-test per day showed that RES animals performed better than $\mathrm{CON}$ animals on the second day of the training phase $(p<0.05)$. The final level of performance on day 3 and the retention trial on day 10 were not different between CON and RES. 


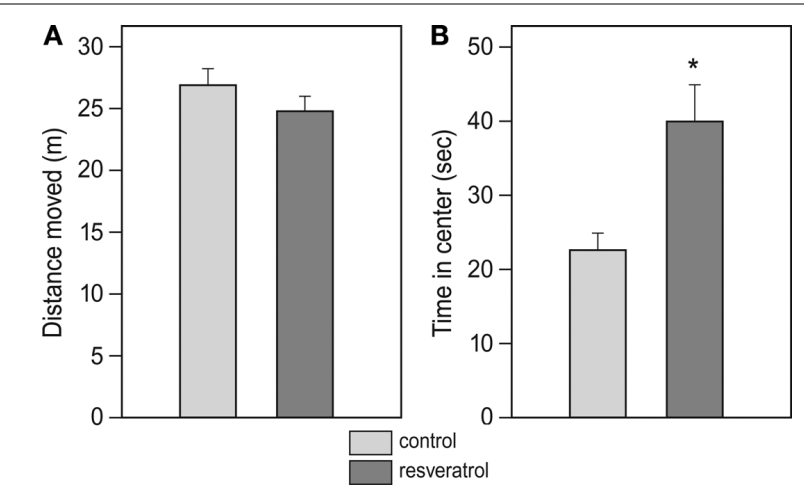

FIGURE 1 | Effects of chronic resveratrol supplementation on locomotor behavior in an open field. The test duration was $5 \mathrm{~min}$ and locomotion of CON $(n=28)$ and RES $(n=28)$ animals was monitored with an automated video analysis system. (A)Total distance covered during the test. (B)Time spent in the center area (diameter: $60 \mathrm{~cm}$ ) of the arena $\left({ }^{*} p=0.003\right.$ ).

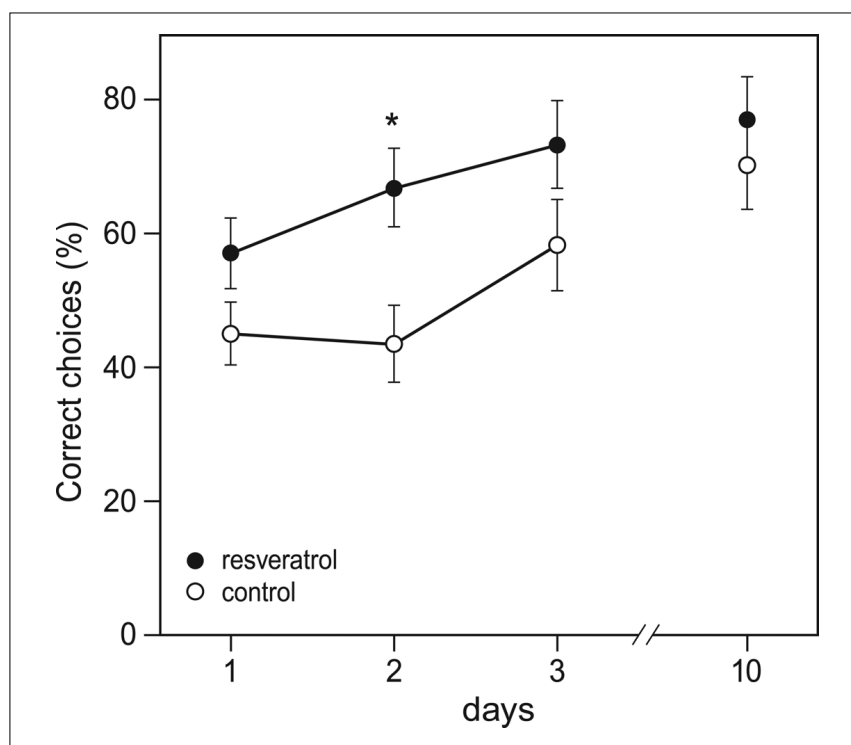

FIGURE 2 | Effects of chronic resveratrol supplementation on Y-maze learning. The Y-maze test consisted of 3 training days and a retention day 1 week later. Each day consisted of eight trials and the performance of the animals is expressed as the percentage of correct choices per day. RES animals ( $n=28$ ) show faster acquisition of the task then CON animals $(n=28)$, as shown on day 2 ( $\left.^{*} p=0.016\right)$.

\section{CHOLINERGIC CELL NUMBERS AND FIBER DENSITY}

Analysis of cholinergic cell number in the nucleus basalis did not reveal any difference between CON $(n=11)$ and RES $(n=11)$, (Figure 3A). Also, cholinergic fiber density in the CA1 and dentate gyrus of the hippocampus and the PARII region of the cortex was unchanged after RES treatment (Figures 3B-D).

\section{CEREBROVASCULAR SYSTEM}

Electron microscopical analysis of brain tissue revealed that vascular density in the hippocampus of RES mice was about 15\% higher than that in the control animals ( $p=0.019$; Figure 4). In the parietal cortex, vascular density was not different between the two groups.
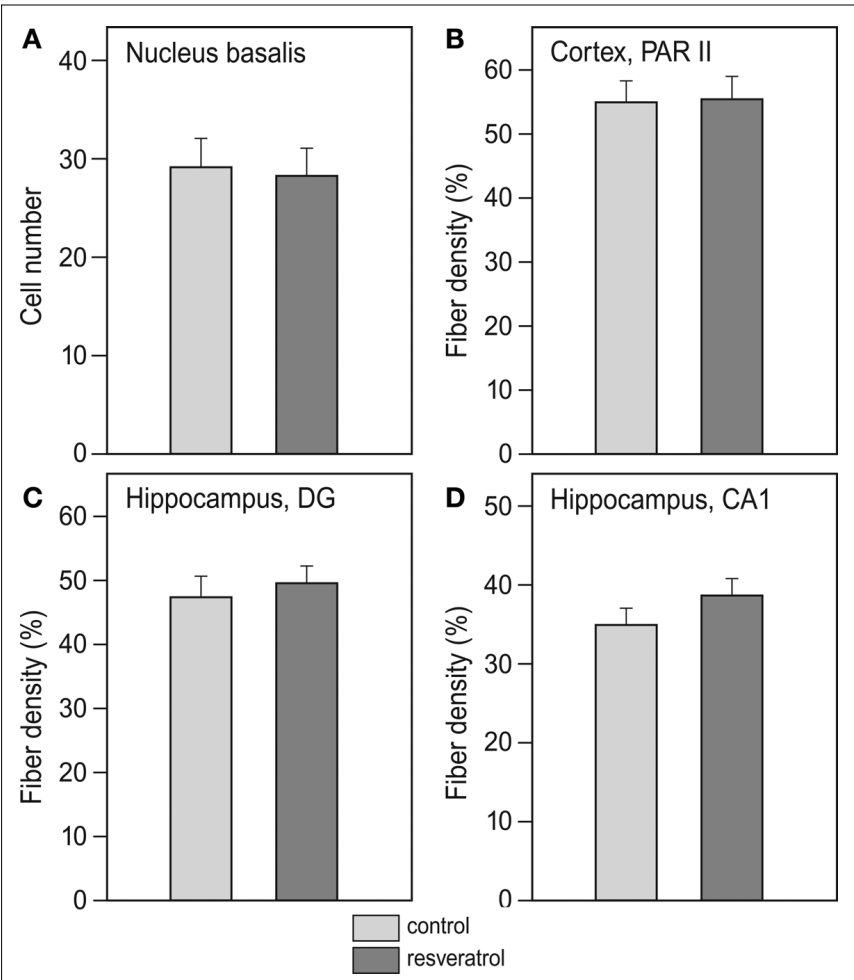

FIGURE 3 | Effects of chronic resveratrol supplementation on the cholinergic system. (A) Average cholinergic cell number per section in the nucleus basalis is unchanged in the resveratrol-treated animals $(\boldsymbol{n}=11)$ versus control-treated animals $(\boldsymbol{n}=11)$. (B) Cholinergic fiber density in hippocampal CA1 and (C) dentate gyrus show no effect of resveratrol treatment. (D) Cholinergic fiber density in the parietal cortex (PARII) does not show an effect of resveratrol treatment.

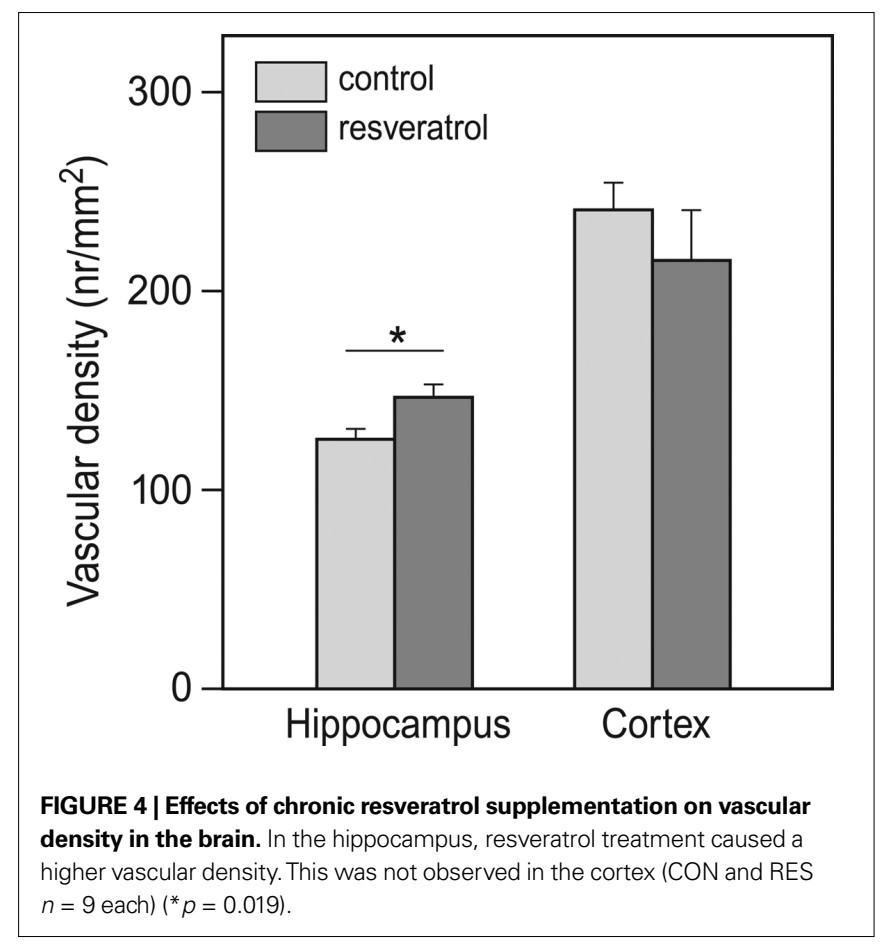


The endothelial cells of cerebral microvessels exhibited two discernible abnormalities. The apical surface of the endothelial cells displayed microvillus-like processes into the lumen (Figure 5A) and empty, large vacuoles formed primarily in the vicinity of tight junctions (Figure 5C). The basement membrane of capillaries occasionally appeared thickened in the form of local exfoliations, while the arteriolar basement membrane, particularly between the endothelial and smooth muscle cells was affected by hyalinosis (Figure 5E). The occurrence of basement membrane thickening and endothelial processes was not
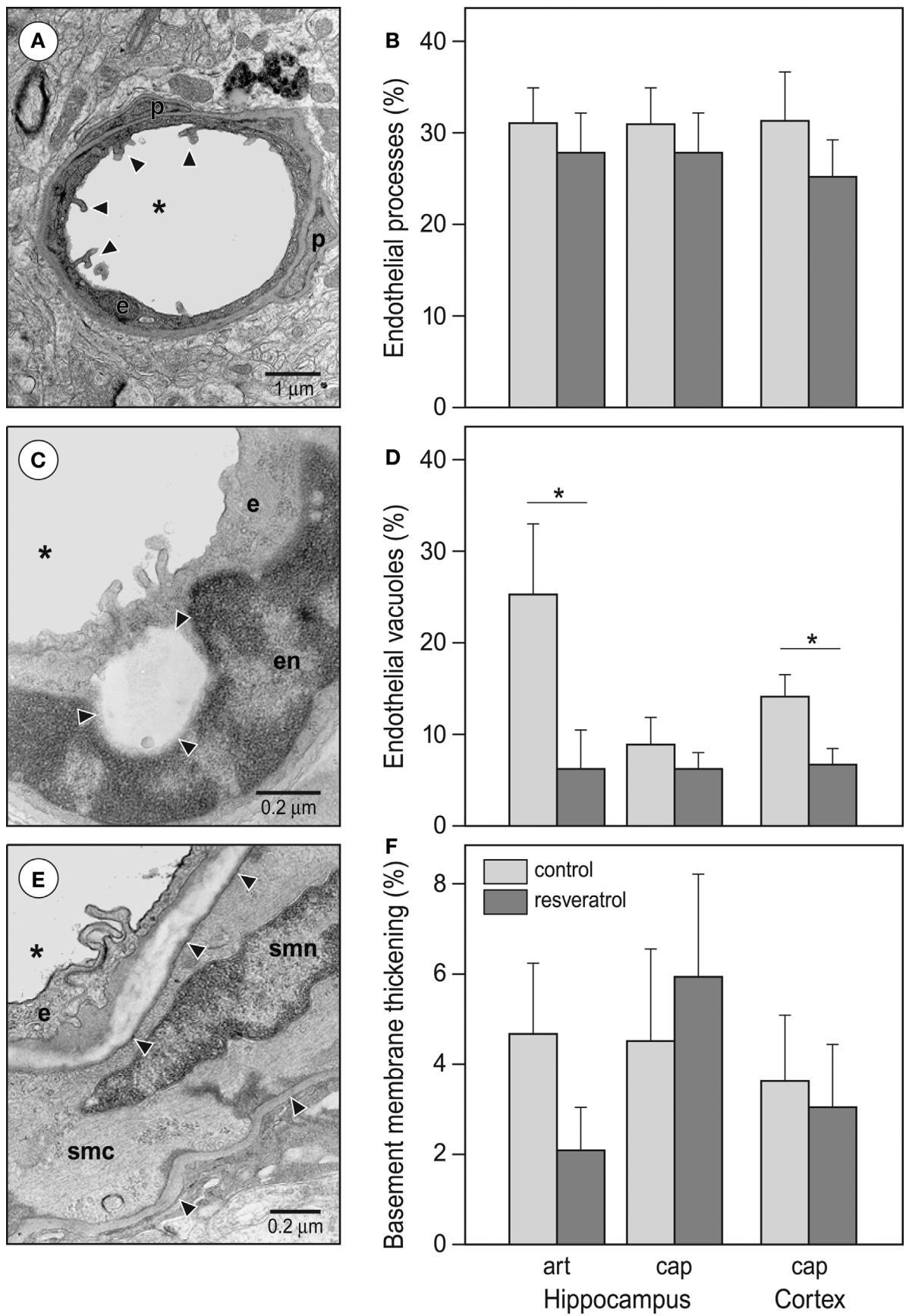

FIGURE 5 | Effects of resveratrol supplementation on the occurrence of microvascular abnormalities. (A) Representative photomicrograph and (B) quantitative analysis of endothelial processes. Arrowheads point at the typical endothelial processes of a cortical capillary. (C) Representative photomicrograph and (D) quantitative analysis of endothelial vacuoles $\left({ }^{*} p<0.05\right)$.

The photomicrograph demonstrates a capillary in the hippocampus CA1 region. Arrowheads are pointing at the large, empty endothelial vacuole.

(E) Representative photomicrograph and (F) quantitative analysis of vascular basement membrane thickening. The photomicrograph demonstrates an arteriole in the hippocampal vascular layer. Arrowheads are pointing at healthy and thickened segments of the basement membrane. Abbreviations: asterisk: microvascular lumen, art: arterioles, cap: capillaries, e: endothelial cell, en: endothelial cell nucleus, p: pericyte, smc: smooth muscle cell, smn: smooth muscle cell nucleus (CON and RES $n=9$ ). 
significantly affected by dietary resveratrol (Figures 5B,F). However, the number of microvessels with endothelial vacuoles was significantly reduced by chronic resveratrol treatment (Figure 5D) in both hippocampal arterioles $(p<0.05)$ and cortical capillaries $(p<0.05)$.

To assess whether cognitive functioning in the aged mice was related to their cerebrovascular status, individual Y-maze performance was correlated with vascular parameters. The Y-maze scores on day 2 and 3 as well as the slope of the learning curve from day 1 to 3 were used as measures of cognitive functioning. Y-maze performance on day 2 correlated positively with capillary density in the cortex, although this only reached statistical significance in the RES group (CON: $R=0.59, p=0.09$; RES: $R=0.86, p=0.03$ ). On the other hand, in the CON animals, Y-maze performance on day 3 showed a negative correlation with the $\%$ of endothelial vacuoles in the cortex (CON: $R=-0.75, p=0.02$; RES: $R=0.36$, $p>0.1$ ), a negative correlation with endothelial processes (CON: $R=-0.77, p=0.04$; RES: $R=-0.19, p>0.1)$ and a negative correlation with basement membrane thickness (CON: $R=-0.88$, $p=0.01$; RES: $R=-0.15, p>0.1$ ) in the hippocampus. Also the slope of the Y-maze learning curve showed a negative correlation with a number of cortical vascular abnormalities like basement membrane thickness in the RES group (RES: $-0.85, p=0.03$ ) and endothelial vacuoles in the CON group (CON: $R=-0.69, p=0.04$ ). Most of these correlations suggest that better learning performance is associated with higher capillary density and lower incidence of vascular abnormalities.

\section{DISCUSSION}

Chronic dietary supplementation with the natural polyphenol resveratrol improved performance of aged mice in the acquisition of a Y-maze task and increased the exploration of the center area of an open-field test. The improvement in cognitive performance as observed in the Y-maze was paralleled by an increased microvascular density in the hippocampus and decreased number of vacuolar abnormalities in both hippocampal and cortical microvascular endothelial cells.

Spatial orientation and memory performance in the Y-maze is at least partly dependent on the hippocampus, a brain area often affected by aging (Erickson and Barnes, 2003; Havekes et al., 2007). We found that resveratrol-treated animals performed better during the acquisition of this task. Although differences in general activity and anxiety are potentially confounding factors in most learning tasks, it is unlikely that this explains the results of the Y-maze test in the present study. First, during the free exploration in the habituation trial before the start of training, resveratrolsupplemented and control mice showed similar levels of explorative activity in terms of arm visits and spontaneous alternations. Second, during the training, all animals entered the maze in every trial and latencies were not different for treated versus untreated animals. Furthermore, although the data from the open-field test show an increased exploration in the center of the arena as opposed to the outer rim, it confirms that there was no effect of resveratrol treatment on general locomotor activity, since the total distance covered by animals was the same for both groups. Taken together, the increased performance of the resveratrol-treated animals during the acquisition phase of the Y-maze task most likely indicates an improved cognitive performance or, alternatively, a reduction in the well-established aging-related decline in cognitive performance (Erickson and Barnes, 2003).

The mechanisms behind resveratrol-induced preservation of cognitive performance during aging are most likely multifactorial. In the present study improved performance in the resveratrolsupplemented group was paralleled by higher hippocampal microvessel density and less aberrant microvessels in both hippocampus and cortex compared to the control group. We also tested for associations between Y-maze performance and cerebrovascular parameters on an individual level by means of a correlative analysis. Clearly, this correlative analysis should be interpreted with caution given the high number of possible associations tested and the risk of type- 1 statistical errors. On the other hand, despite the restricted number of animals included in the EM study and the reasonably low variation in the various measures, it is striking that all significant correlations point in the same direction, that is, better performance in the Y-maze was associated with higher capillary density and a lower incidence of vascular abnormalities in either cortex or hippocampus. These findings thus support the idea that beneficial effects of resveratrol on cognitive function are mediated via the cerebrovascular system. A higher microvascular density and increased cerebral blood flow might improve performance directly by increasing glucose and oxygen supply to relevant brain areas (Gold, 2005; Zlokovic, 2008). Additionally, microvessels potentially provide a source of trophic factors such as insulin-like growth factor (IGF)-1 and nerve growth factor that support neuronal function. These factors have been shown to decrease in aging together with reductions in cortical microvascular density (Sonntag et al., 1997) and microvascular growth (Ingraham et al., 2008), and are also implicated in age-related cognitive decline (Ramsey et al., 2004).

An increased microvascular density can be explained in two ways. First, resveratrol could actively stimulate angiogenesis or second, resveratrol could prevent an age-related decline in angiogenesis and/or deterioration of vascular status. Since resveratrol has been reported to inhibit angiogenesis in vitro (Igura et al., 2001; Cao et al., 2004), it seems more likely that resveratrol supplementation in the present study attenuated the age-related decline in vascular density and maintained normal levels of angiogenesis as opposed to an increase in angiogenesis and vessel density over control levels. Moreover, resveratrol appeared to prevent or delay the occurrence of microvascular abnormalities, another hallmark of aging-related decline in the microvascular condition.

There are multiple pathways via which resveratrol could prevent age-related decline in vascular density and occurrence of microvascular abnormalities. First, resveratrols' antioxidative properties prevent oxidation of membrane lipids and ensure normal cellular functioning, whereas excessive oxidative stress has been reported to impair angiogenesis (Urbich et al., 2002; Michaud et al., 2006). Second, resveratrol is likely to influence cerebrovascular health by its capability to reduce platelet aggregation (Bertelli et al., 1995; Stef et al., 2006) and its facilitation of endothelium-dependent vascular relaxation (Rush et al., 2007). Finally, resveratrol may be able to prevent the aging-related decline in cerebrovascular condition via the activation of biochemical pathways that involve SIRT1 (a sirtuin gene encoding a NAD+ dependent deacetylase). Resveratrol has been categorized as a sirtuin-activating compound 
which is known to mimic caloric restriction and was found to increase life span in lower organisms via the activation of sir2, a conserved deacetylase proposed to underlie the beneficial effects of caloric restriction (Howitz et al., 2003; Wood et al., 2004). In mammalian cells, resveratrol and/or resveratrol-activated SIRT1 have been found to induce processes that promote cellular functioning and control cell death (Picard et al., 2004; Chen et al., 2005; Kolthur-Seetharam et al., 2006; Alvira et al., 2007; Okawara et al., 2007; Barger et al., 2008; Pearson et al., 2008; Raval et al., 2008). The finding that caloric restriction itself benefits cortical microvascular density and local cerebral blood flow in aged rats (Lynch et al., 1999) supports the idea of this pathway as a potential mechanism of action. Additionally, both resveratrol and SIRT1 were shown to exert vasoprotective effects against oxidative stress and inflammatory factors (Csiszar et al., 2008).

More recent studies found compelling effects of resveratrol, as a substance that potentially enhances the health and lifespan of organisms. For instance, Barger et al. found that even a low dose of resveratrol partially mimics caloric restriction in mice by assessing transcription patterns in several tissues, including the brain. Resveratrol supplementation was around $25 \%$ of the dosage used in the current study. Interestingly, chronic resveratrol supplementation in mice on a high-calorie diet showed prolonged survival and a prolonged general health (Baur et al., 2006). In this particular study, mice received resveratrol in a chronic fashion, doses and timing highly comparable to our experimental design. The authors found that resveratrol produces changes associated with a longer lifespan, resulting in a physiological shift of middleaged mice on a high-calorie diet towards that of mice on a standard diet. In a more recent study by the same group, resveratrol had no effects on survival in normal mice, but they did find that resveratrol mimics transcriptional patterns in different tissues (Pearson et al., 2008). Although we did not assess survival, but focused on neurobiological function and cerebrovascular condition, our data suggest that one of the target-systems for resveratrol to exert its beneficial effects can be found in a preservation of the cerebrovascular system.

Interestingly, the effects of resveratrol appear to be the most prominent in the hippocampus as reflected by the region-specific increase in microvascular density. As an interesting parallel, the beneficial effects of physical exercise on the microvasculature, have also shown a high degree of regional specificity, even within the hippocampal formation (Pereira et al., 2007; Van der Borght et al., 2009). This demonstrates that, even though the hippocampus

\section{REFERENCES}

Alvira, D., Yeste-Velasco, M., Folch, J., Verdaguer, E., Canudas, A. M., Pallas, M., and Camins, A. (2007). Comparative analysis of the effects of resveratrol in two apoptotic models: inhibition of complex I and potassium deprivation in cerebellar neurons. Neuroscience 147, 746-756.

Amenta, F., Ferrante, F., Mancini, M., Sabbatini, M., Vega, J. A., and Zaccheo, D. (1995). Effect of long-term treatment with the dihydropyridine-type calcium channel blocker darodipine

is one of the most vulnerable regions when it comes to age-related pathology, the hippocampal microvasculature contains adaptive potential and may prove to be an interesting therapeutic target.

In contrast to the results on the cerebrovascular level, we did not find any effects of chronic resveratrol treatment on structural parameters of the cholinergic system i.e. cholinergic cell number and fiber density. The lack of effects on neuronal level is actually paralleled by previous studies on aging, where no quantitative difference in the number of neurons or synapses were found (for review see Coleman and Flood, 1987). Furthermore, previous studies on dietary supplementation in Tg2576 transgenic mice, a model for Alzheimer's disease, found a strong effect on spatial learning, but no effects on histological read outs (Stackman et al., 2003; Quinn et al., 2007). A recent study, however, argues in favor of the direct effects of resveratrol on neuronal health since they showed a reduction in neurodegeneration (cell death) in parallel with a rescued cognitive functioning after resveratrol treatment (Kim et al., 2007). However, this study used intracerebroventricular injections which leads to a direct treatment of neuronal tissue. When applied in an oral fashion, resveratrol is required to enter the brain in sufficient levels in order to exhibit its direct effects on neuronal health. In the present study we show that resveratrol is present in the blood, fluctuating according to daily food intake. However, the bioavailability of resveratrol and relative concentrations in different tissues are still under discussion (Rossi et al., 2008) and it is unknown whether sufficient concentrations of resveratrol cross the blood-brain barrier to exert direct beneficial effects at the neuronal level. This should be considered when evaluating the relevance of the physiological effects of resveratrol in view of treatment options.

In conclusion, this is the first study to show beneficial effects of long-term dietary supplementation with the natural polyphenol resveratrol on cognitive functioning and cerebrovascular condition. The status of the cerebrovascular system and the neurovascular unit have gained increasing interest as a potential target for treatment of aging associated disorders of the brain (Iadecola, 2004; Zlokovic, 2008). In this light, we propose that ingestion of resveratrol, thereby targeting the integrity of the cerebrovascular system, can be an effective and simple method to support healthy aging of the brain.

\section{ACKNOWLEDGMENTS}

We thank Gerrit Witte and Martijn de Wilde at Danone Research for determining resveratrol levels in the blood samples. We thank Jan Keijser of the Department for Molecular Neurobiology in Haren for his assistance in the Quantimet analysis.

Baur, J. A., Pearson, K. J., Price, N. L., Jamieson, H. A., Lerin, C., Kalra, A., Prabhu, V. V., Allard, J. S., Lopez-Lluch, G., Lewis, K., Pistell, P. J., Poosala, S. Becker, K. G., Boss, O., Gwinn, D., Wang, M., Ramaswamy, S., Fishbein, K. W., Spencer, R. G., Lakatta,E. G., Le Couteur, D., Shaw, R. J., Navas, P., Puigserver, P., Ingram, D. K., de Cabo, R., and Sinclair, D.A.(2006). Resveratrolimproves health and survival of mice on a high-calorie diet. Nature 444, 337-342

Baur, J. A., and Sinclair, D. A. (2006). Therapeutic potential of resveratrol: the in vivo evidence. Nat. Rev. Drug Discov. 5, 493-506.

Bertelli, A. A., Giovannini, L., Giannessi, D., Migliori, M., Bernini, W., Fregoni, M., and Bertelli,A. (1995). Antiplatelet activity of synthetic and natural resveratrol in red wine. Int. J. Tissue React. 17, 1-3.

Buee,L., Hof,P.R., Bouras, C., Delacourte,A., Perl, D. P., Morrison, J. H., and Fillit, H. M. (1994). Pathologicalalterations of the cerebral microvasculature in Alzheimer's disease and related dementing disorders. Acta Neuropathol. 87, 469-480. 
Burns, E. M., Kruckeberg, T. W., and Gaetano, P. K. (1981). Changes with age in cerebral capillary morphology. Neurobiol. Aging 2, 283-291.

Cao, Z., Fang, J., Xia, C., Shi, X., and Jiang, B. H. (2004). trans-3,4,5'Trihydroxystibene inhibits hypoxiainducible factor lalpha and vascular endothelial growth factor expression in human ovarian cancer cells. Clin. Cancer Res. 10, 5253-5263.

Chen, J., Zhou, Y., Mueller-Steiner, S., Chen, L. F., Kwon, H., Yi, S., Mucke, L., and Gan, L. (2005). SIRT1 protects against microglia-dependent amyloidbeta toxicity through inhibiting NFkappaB signaling. J. Biol. Chem. 280, 40364-40374.

Coleman, P. D., and Flood, D. G. (1987). Neuron numbers and dendritic extent in normal aging and Alzheimer's disease. Neurobiol. Aging 8, 521-545.

Csiszar, A., Labinskyy, N., Podlutsky, A., Kaminski, P. M., Wolin, M. S., Zhang, C., Mukhopadhyay, P., Pacher, P., Hu, F., de Cabo, R., Ballabh, P., and Ungvari, Z. (2008). Vasoprotective effects of resveratrol and SIRT1: attenuation of cigarette smoke-induced oxidative stress and proinflammatory phenotypic alterations. Am. J. Physiol. Heart Circ. Physiol. 294, H2721-2735.

Das, S., Alagappan, V. K., Bagchi, D., Sharma, H. S., Maulik, N., and Das, D. K. (2005). Coordinated induction of iNOS-VEGF-KDR-eNOS after resveratrol consumption: a potential mechanism for resveratrol preconditioning of the heart. Vascul. Pharmacol. 42, 281-289.

De Jong, G. I., De Vos, R. A., Steur, E. N., and Luiten, P. G. (1997). Cerebrovascular hypoperfusion: a risk factor for Alzheimer's disease? Animal model and postmortem human studies. Ann. N. Y. Acad. Sci. 826, 56-74.

de Wilde, M. C., Farkas, E., Gerrits, M., Kiliaan, A. J., and Luiten, P. G. (2002). The effect of n-3 polyunsaturated fatty acid-rich diets on cognitive and cerebrovascular parameters in chronic cerebral hypoperfusion. Brain Res. 947, 166-173.

Dong, W., Li, N., Gao, D., Zhen, H., Zhang, X., and Li, F. (2008). Resveratrol attenuates ischemic brain damage in the delayed phase after stroke and induces messenger RNA and protein express for angiogenic factors. J. Vasc. Surg. 48, 709-714.

Erickson, C. A., and Barnes, C. A. (2003). The neurobiology of memory changes in normal aging. Exp. Gerontol. 38, 61-69.

Farkas, E., De Vos, R. A., Jansen Steur, E. N., and Luiten, P. G. (2000). Are Alzheimer's disease, hypertension, and cerebrocapillary damage related? Neurobiol. Aging 21, 235-243.
Farkas, E., and Luiten, P. G. (2001). Cerebral microvascular pathology in aging and Alzheimer's disease. Prog. Neurobiol. 64, 575-611.

Farkas, E., Luiten, P. G., and Bari, F. (2007). Permanent, bilateral common carotid artery occlusion in the rat: a model for chronic cerebral hypoperfusion-related neurodegenerative diseases. Brain Res. Rev. 54, 162-180.

Farkas, I. G., Czigner, A., Farkas, E., Dobo, E., Soos, K., Penke, B., Endresz, V., and Mihaly, A. (2003). Beta-amyloid peptide-induced blood-brain barrier disruption facilitates T-cell entry into the rat brain. Acta Histochem. 105, 115-125.

Fitzpatrick, D. F., Hirschfield, S. L., and Coffey, R. G. (1993). Endotheliumdependent vasorelaxing activity of wine and other grape products. Am. J. Physiol. 265, H774-778.

Frankel, E. N., Waterhouse, A. L., and Kinsella, J. E. (1993). Inhibition of human LDL oxidation by resveratrol. Lancet 341, 1103-1104.

Fremont, L. (2000). Biological effects of resveratrol. Life Sci. 66, 663-673.

Fremont, L., Belguendouz, L., and Delpal, S. (1999). Antioxidant activity of resveratrol and alcohol-free wine polyphenols related to LDL oxidation and polyunsaturated fatty acids. Life Sci. 64, 2511-2521.

Gehm,B.D., McAndrews, J.M., Chien, P.Y., and Jameson, J. L. (1997). Resveratrol, a polyphenolic compound found in grapes and wine, is an agonist for the estrogen receptor. Proc. Natl. Acad. Sci. U.S.A. 94, 14138-14143.

Gold, P.E. (2005). Glucose and age-related changes in memory. Neurobiol. Aging 26(Suppl. 1), 60-64.

Havekes, R., Timmer, M., and Van der Zee, E. A. (2007). Regional differences in hippocampal PKA immunoreactivity after training and reversal training in a spatial Y-maze task. Hippocampus 17, 338-348.

Hedreen, J. C., Bacon, S. J., and Price, D. L. (1985). A modified histochemical technique to visualize acetylcholinesterase-containing axons. J. Histochem. Cytochem. 33, 134-140.

Howitz, K. T., Bitterman, K. J., Cohen, H. Y., Lamming, D. W., Lavu, S., Wood, J. G., Zipkin, R. E., Chung, P., Kisielewski, A., Zhang, L. L., Scherer, B., and Sinclair, D. A. (2003). Small molecule activators of sirtuins extend Saccharomyces cerevisiae lifespan. Nature 425, 191-196.

Iadecola, C. (2004). Neurovascular regulation in the normal brain and in Alzheimer's disease. Nat. Rev. Neurosci. 5, 347-360.

Igura, K., Ohta, T., Kuroda, Y., and Kaji, K. (2001). Resveratrol and quercetin inhibit angiogenesis in vitro. Cancer Lett. 171, 11-16.

Ingraham, J. P., Forbes, M. E., Riddle, D. R., and Sonntag, W. E. (2008). Aging reduces hypoxia-induced microvascular growth in the rodent hippocampus. J. Gerontol. A Biol. Sci. Med. Sci. 63, 12-20.

Jager, U., and Nguyen-Duong, H. (1999). Relaxant effect of trans-resveratrol on isolated porcine coronary arteries. Arzneimittelforschung 49, 207-211.

Jang, J. H. and Surh, Y. J. (2003). Protective effect of resveratrol on beta-amyloid-induced oxidative PC12 cell death. Free Radic. Biol. Med. 34, 1100-1110.

Jang, M., Cai, L., Udeani, G. O., Slowing, K. V., Thomas, C. F., Beecher, C. W., Fong, H. H., Farnsworth, N. R., Kinghorn, A. D., Mehta, R. G., Moon, R. C., and Pezzuto, J. M. (1997). Cancer chemopreventive activity of resveratrol, a natural product derived from grapes. Science 275, 218-220.

Keuker, J. I., Luiten, P. G., and Fuchs, E. (2000). Capillary changes in hippocampal CA 1 and $\mathrm{CA} 3$ areas of the aging rhesus monkey. Acta Neuropathol. 100 , 665-672.

Kim, D., Nguyen, M. D., Dobbin, M. M., Fischer, A., Sananbenesi, F., Rodgers, J. T., Delalle, I., Baur, J. A., Sui, G., Armour, S. M., Puigserver, P., Sinclair, D. A., and Tsai, L. H. (2007). SIRT1 deacetylase protects against neurodegeneration in models for Alzheimer's disease and amyotrophic lateral sclerosis. EMBO J. 26, 3169-3179.

Kolthur-Seetharam, U., Dantzer, F., McBurney, M. W., de Murcia, G., and Sassone-Corsi, P. (2006). Control of AIF-mediated cell death by the functional interplay of SIRT1 and PARP-1 in response to DNA damage. Cell Cycle 5, 873-877.

Kopp, P. (1998). Resveratrol, a phytoestrogen found in red wine. A possible explanation for the conundrum of the 'French paradox'? Eur. J. Endocrinol. 138, 619-620.

Labinskyy, N., Csiszar, A., Veress, G., Stef, G., Pacher, P., Oroszi, G., Wu, J., and Ungvari, Z. (2006). Vascular dysfunction in aging: potential effects of resveratrol, an anti-inflammatory phytoestrogen. Curr. Med. Chem. 13, 989-996.

Lee, E. Y., Lee, S. Y., Lee, T. S., Chi, J. G., Choi, W., and Suh, Y. H. (2000). Ultrastructural changes in microvessel with age in the hippocampus of senescence-accelerated mouse (SAM)P/10. Exp. Aging Res. 26, 3-14.

Lu, K. T., Chiou, R.Y., Chen, L. G., Chen, M. H., Tseng, W. T., Hsieh, H. T., and Yang, Y. L. (2006). Neuroprotective effects of resveratrol on cerebral ischemiainduced neuron loss mediated by free radical scavenging and cerebral blood flow elevation. J. Agric. Food Chem. 54, 3126-3131.

Lynch, C. D., Cooney, P. T., Bennett, S. A., Thornton, P. L., Khan, A. S., Ingram, R. L., and Sonntag, W. E. (1999). Effects of moderate caloric restriction on cortical microvascular density and local cerebral blood flow in aged rats. Neurobiol. Aging 20, 191-200.

Michaud, S. E., Dussault, S., Groleau, J., Haddad, P., and Rivard, A. (2006). Cigarette smoke exposure impairs VEGF-induced endothelial cell migration: role of $\mathrm{NO}$ and reactive oxygen species. J. Mol. Cell. Cardiol. 41, 275-284.

Mizutani, K., Ikeda, K., Kawai, Y., and Yamori, Y. (2001). Protective effect of resveratrol on oxidative damage in male and female stroke-prone spontaneously hypertensive rats. Clin. Exp. Pharmacol. Physiol. 28, 55-59.

Okawara, M., Katsuki, H., Kurimoto, E., Shibata, H., Kume, T., and Akaike, A. (2007). Resveratrol protects dopaminergic neurons in midbrain slice culture from multiple insults. Biochem. Pharmacol. 73, 550-560.

Pearson, K. J., Baur, J. A., Lewis, K. N., Peshkin, L., Price, N. L., Labinskyy, N., Swindell, W. R., Kamara, D., Minor, R. K., Perez, E., Jamieson, H. A., Zhang, Y., Dunn, S. R., Sharma, K., Pleshko, N., Woollett, L. A., Csiszar, A., Ikeno, Y., Le Couteur, D., Elliott, P. J., Becker, K. G., Navas, P., Ingram, D. K., Wolf, N. S., Ungvari, Z., Sinclair, D. A., and de Cabo, R. (2008). Resveratrol delays age-related deterioration and mimics transcriptional aspects of dietary restriction without extending life span. Cell Metab. 8, 157-168.

Pereira, A. C., Huddleston, D. E., Brickman, A. M., Sosunov, A. A., Hen, R., McKhann, G. M., Sloan, R. Gage, F. H., Brown, T. R., and Small, S. A. (2007). An in vivo correlate of exercise-induced neurogenesis in the adult dentate gyrus. Proc. Natl. Acad. Sci. U.S.A. 104, 5638-5643.

Picard, F., Kurtev, M., Chung, N., ToparkNgarm, A., Senawong, T., Machado De Oliveira, R., Leid, M., McBurney, M. W., and Guarente, L. (2004). Sirtl promotes fat mobilization in white adipocytes by repressing PPAR-gamma. Nature 429, 771-776.

Quinn, J. F., Bussiere, J. R., Hammond, R. S., Montine, T. J., Henson, E., Jones, R. E. and Stackman, R. W. Jr. (2007). Chronic dietary alpha-lipoic acid reduces deficits in hippocampal memory of aged Tg2576 mice. Neurobiol. Aging 28, 213-225.

Ramsey, M. M., Weiner, J. L., Moore, T. P., Carter, C. S., and Sonntag, W. E. (2004). Growth hormone treatment 
attenuates age-related changes in hippocampal short-term plasticity and spatial learning. Neuroscience 129, 119-127.

Raval,A.P., Lin, H.W., Dave, K. R., Defazio, R.A., Morte, D. D., Kim, E. J., and PerezPinzon, M. A. (2008). Resveratrol and ischemic preconditioning in the brain. Curr. Med. Chem. 15, 1545-1551.

Renaud, S., and de Lorgeril, M. (1992). Wine, alcohol, platelets, and the French paradox for coronary heart disease. Lancet 339, 1523-1526.

Riddle, D. R., Sonntag, W. E., and Lichtenwalner, R. J. (2003). Microvascular plasticity in aging. Ageing Res. Rev. 2, 149-168.

Rossi, L., Mazzitelli, S., Arciello, M., Capo, C. R., and Rotilio, G. (2008). Benefits from dietary polyphenols for brain aging and Alzheimer's disease. Neurochem. Res. 33, 2390-2400.

Rush, J. W., Quadrilatero, J., Levy, A. S. and Ford, R. J. (2007). Chronic resveratrol enhances endothelium-dependent relaxation but does not alter eNOS levels in aorta of spontaneously hypertensive rats. Exp. Biol. Med. 232, 814-822.

Sonntag, W. E., Lynch, C., Thornton, P., Khan, A., Bennett, S., and Ingram, R. (2000). The effects of growth hormone and IGF-1 deficiency on cerebrovascular and brain ageing. $J$. Anat. 197(Pt 4) 575-585.
Sonntag, W.E., Lynch, C. D., Cooney, P. T., and Hutchins, P. M. (1997). Decreases in cerebral microvasculature with age are associated with the decline in growth hormone and insulin-like growth factor 1. Endocrinology 138, 3515-3520.

Stackman, R. W., Eckenstein, F., Frei, B., Kulhanek, D., Nowlin, J., and Quinn, J. F. (2003). Prevention of age-related spatial memory deficits in a transgenic mouse model of Alzheimer's disease by chronic Ginkgo biloba treatment. Exp. Neurol. 184, 510-520.

Stef, G., Csiszar, A., Lerea, K., Ungvari, Z., and Veress, G. (2006). Resveratrol inhibits aggregation of platelets from high-risk cardiac patients with aspirin resistance. J. Cardiovasc. Pharmacol. $48,1-5$.

Urbich, C., Dernbach, E., Aicher, A., Zeiher,A.M., and Dimmeler, S. (2002). CD40 ligand inhibits endothelial cell migration by increasing production of endothelial reactive oxygen species. Circulation 106, 981-986.

Van der Borght, K., Kobor-Nyakas, D. E., Klauke, K., Eggen, B. J., Nyakas, C., Van der Zee, E. A., and Meerlo, P. (2009). Physical exercise leads to rapid adaptations in hippocampal vasculature: temporal dynamics and relationship to cell proliferation and neurogenesis. Hippocampus 19, 928-936.
Virgili, M., and Contestabile, A. (2000). Partial neuroprotection of in vivo excitotoxic brain damage by chronic administration of the red wine antioxidant agent, trans-resveratrol in rats. Neurosci. Lett. 281, 123-126.

Wang, Q., Xu, J., Rottinghaus, G. E., Simonyi, A., Lubahn, D., Sun, G. Y., and Sun, A. Y. (2002). Resveratrol protects against global cerebral ischemic injury in gerbils. Brain Res. 958, 439-447.

Wood, J.G., Rogina, B., Lavu, S., Howitz, K., Helfand, S. L., Tatar, M., and Sinclair, D. (2004). Sirtuin activators mimic caloric restriction and delay ageing in metazoans. Nature 430, 686-689.

Wu, J.M., Wang, Z. R., Hsieh, T.C., Bruder, J. L., Zou, J. G. and Huang, Y.Z. (2001). Mechanism of cardioprotection by resveratrol, a phenolic antioxidant present in red wine (Review). Int. J. Mol. Med. 8, 3-17.

Zhang, H., Schools, G. P., Lei, T., Wang, W. Kimelberg, H. K., and Zhou,M. (2008). Resveratrol attenuates early pyramidal neuron excitability impairment and death in acute rat hippocampal slices caused by oxygen-glucose deprivation. Exp. Neurol. 212, 44-52.

Zlokovic, B. V. (2008). The bloodbrain barrier in health and chronic neurodegenerative disorders. Neuron 57, 178-201.
Conflict of Interest Statement: The research conducted in the paper was financially supported by Danone Research, Centre for Specialised nutrition. Danone Research has a financial interest in the component resveratrol. Eline van der Beek is employed at Danone Research, Centre for Specialised Nutrition in Wageningen, the Netherlands. Charlotte Oomen, Eszter Farkas, Viktor Roman, Paul Luiten and Peter Meerlo, have no actual or potential conflict of interests concerning the research presented in the paper.

Received: 31 July 2009; paper pending published: 10 October 2009; accepted: 23 November 2009; published online: 09 December 2009.

Citation: Oomen CA, Farkas E, Roman V, van der Beek EM, Luiten PGM and Meerlo $P$ (2009) Resveratrol preserves cerebrovascular density and cognitive function in aging mice. Front. Ag. Neurosci. 1:4. doi: 10.3389/neuro.24.004.2009

Copyright (c) 2009 Oomen, Farkas, Roman, van der Beek, Luiten and Meerlo. This is an open-access article subject to an exclusive license agreement between the authors and the Frontiers Research Foundation, which permits unrestricted use, distribution, and reproduction in any medium, provided the original authors and source are credited. 